\title{
A node-disjoint multi-path routing protocol based on location prediction for mobile ad hoc networks
}

\author{
Natarajan Meghanathan
}

Department of Computer Science, Jackson State University, Jackson, MS, USA

E-mail: natarajan.meghanathan@jsums.edu, Tel +1-601-979-3661, Fax.+1-601-979-2478

\begin{abstract}
We propose a node-disjoint multi-path version to the location prediction-based routing protocol (LPBR-M) for mobile ad hoc networks to simultaneously reduce the number of global broadcast multi-path route discoveries and the average hop count per path from the source to the destination. During a global broadcast route discovery, the intermediate forwarding nodes include their location and mobility information in the Route-Request (MP-RREQ) messages. The destination node selects a set of nodedisjoint paths from the MP-RREQ messages received and sends a sequence of Route-Reply (MP-RREP) messages on each of the node-disjoint paths extracted. Upon failure of all the node-disjoint paths, the destination attempts to locally construct a global topology (using the location and mobility information collected during the global broadcast route discovery) and sends a sequence of MP-LPBR-RREP messages on each of the node-disjoint paths on the predicted global topology. If the source receives at least one MP-LPBR-RREP message within a certain time, it continues to send the data packets along the newly learnt node-disjoint paths. Otherwise, the source initiates another global broadcast route discovery. Simulation results indicate that LPBR-M incurs the longest time between successive route discoveries, lowest energy consumed per data packet and the lowest control message overhead.
\end{abstract}

Keywords: Multi-path Routing Protocol, Mobile Ad hoc Networks, Location Prediction, Node Disjoint, Simulation

\section{Introduction}

On-demand routing protocols for mobile ad hoc networks (MANETs) incur high route discovery latency and also incur frequent route discoveries in the presence of a dynamically changing topology. Recent research has started to focus on multi-path routing protocols for fault tolerance and load balancing. Multi-path on-demand routing protocols tend to compute multiple paths, at both the traffic sources as well as at intermediary nodes, in a single route discovery attempt. This reduces both the route discovery latency and the control overheads as a route discovery is needed only when all the discovered paths fail. Spreading the traffic along several routes could alleviate congestion and bottlenecks. Multi-path routing also provides a higher aggregate bandwidth and effective load balancing as the data forwarding load can be distributed over all the paths.

Multi-paths can be of two types: link-disjoint and node-disjoint. For a given source $s$ and destination $d$, the set of link-disjoint $s-$ $d$ routes comprises of paths that have no link present in more than one constituent $s-d$ path. Similarly, the set of node-disjoint $s-d$ routes comprises of paths that have no node (other than the source and destination) present in more than one constituent $s-d$ path. Multi-path routing protocols proposed for ad hoc networks make use of the propagation of the Route-Request (RREQ) messages along several paths to the destination and let the destination to send Route-Reply (RREP) along more than one path. The routing protocols avoid the RREP storm by selecting only few of the different paths. Since nodes communicate through the shared wireless medium, the selected paths need to be as independent as possible in order to avoid transmissions from a node along one path interfering with transmissions on a different path. The aggregate bandwidth achieved with multi-path routing may not be the sum of the bandwidth of the individual paths. Metrics such as correlation factor and coupling factor are used to calculate the relative degree of independence among the multiple paths (Mueller et al., 2004). The correlation factor, measured only for node-disjoint paths, indicates the number of links connecting two node-disjoint paths. The coupling factor, measured for both node-disjoint and 
link-disjoint paths, is defined as the average number of nodes that are blocked from receiving data on one of the paths when a node in the other path is transmitting. Node-disjoint routes offer the highest degree of fault tolerance and aggregate bandwidth.

Xu et al. (2005) advocate the need to consider similarity among the multiple $s-d$ paths with that of the shortest $s-d$ path and stress the need to use similar paths for multi-path data propagation. Routing using multiple paths similar to the shortest path will reduce the chances of out-of-order packet delivery and also result in lower end-to-end delay per packet. Wang et al. (2005) developed an analytical model for evaluating the effectiveness of multi-path routing. They showed that unless we use a very large number of paths, the load distribution with multi-path routing is almost the same as in single path routing.

Most of the multi-path routing protocols proposed in the literature are either extensions of the Dynamic Source Routing (DSR) protocol (Johnson et al., 2000) or the Ad hoc On-demand Distance Vector (AODV) routing protocol (Perkins and Royer, 1999). The multi-path routing protocols that are currently being reviewed include: (i) Split multi-path routing (SMR) protocol (Lee and Gerla, 2001), an extension of DSR; (ii) Ad hoc On-demand Multi-path Distance Vector (AOMDV) routing protocol (Marina and Das, 2001), an extension of AODV to compute multiple loop-free link-disjoint routes; (iii) AODV-Multi-path (AODVM) routing protocol (Ye et al., 2003), an extension of the AODV protocol to determine node-disjoint routes; (iv) Geographic Multi-path Routing Protocol (Loscri and Marano, 2006) proposed to reduce interference due to route coupling and (v) Energy-aware Multipath Routing Protocol ( $\mathrm{Li}$ et al., 2005) that considers the available energy and the forwarding load at the intermediate nodes of the multiple paths before distributing the load across them.

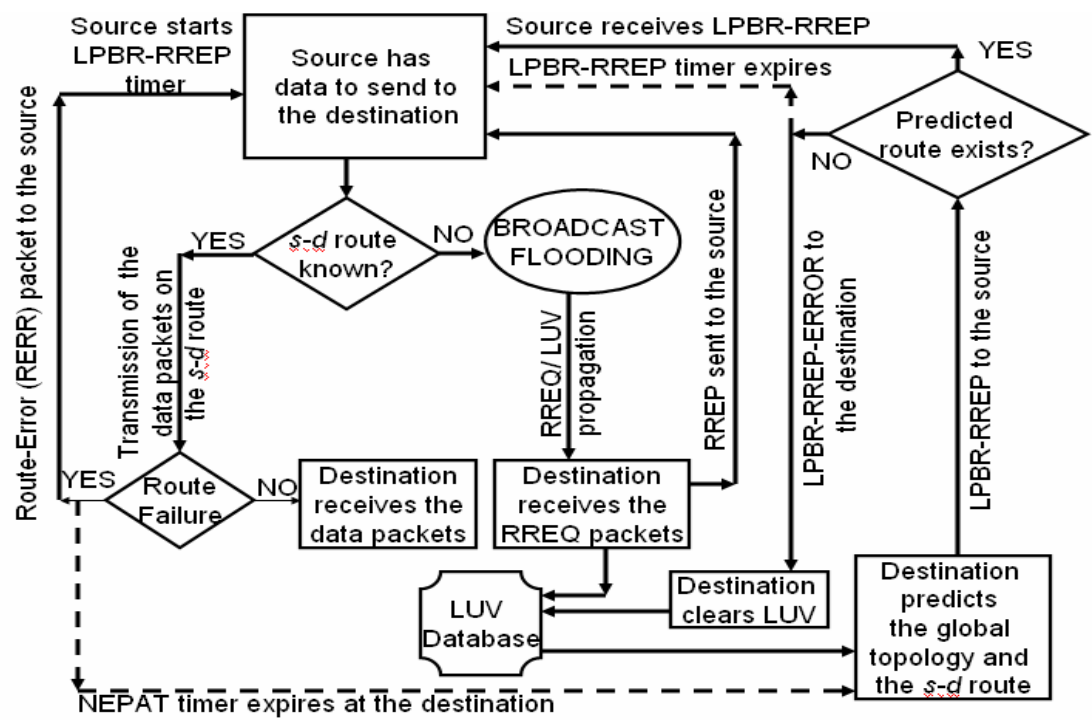

Figure 1. Flowchart Illustrating the Working of the Location Prediction Based Routing (LPBR) Protocol

Meghanathan (2009a) developed a location prediction based routing (LPBR) protocol for single-path unicast routing in MANETs. LPBR attempts to simultaneously reduce the number of global broadcast route discoveries as well as the hop count of the paths for a source-destination session. LPBR works as follows: During a regular broadcast route discovery, LPBR collects the location and mobility information of the nodes in the network in the form of Location Update Vectors (LUVs) and stores the LUVs at the destination node of the route search process. When the minimum-hop route discovered through the broadcast route discovery fails, the destination node attempts to predict the current location of each node using the location and mobility information collected during the latest broadcast route discovery. A minimum hop path Dijkstra algorithm (Cormen et al., 2001) is run on the locally predicted global topology. If the predicted minimum hop route exists in reality, no expensive broadcast route discovery is needed and the source continues to send data packets on the discovered route; otherwise, the source initiates another broadcast route discovery. Figure 1 illustrates the working of the LPBR protocol.

In this paper, we develop a node-disjoint multi-path extension to the LPBR protocol, referred to as LPBR-M. Meghanathan (2007) observed that for different conditions of network density and node mobility, the number of broadcast route discoveries needed for node-disjoint multi-path routing is not significantly different from the number of route discoveries for link-disjoint multi-path routing. Also, there is no much difference in the average hop count of the node-disjoint paths and the link-disjoint paths. On the other hand, node-disjoint paths are preferred for fault tolerance, load balancing and extending the lifetime of the nodes. LPBR-M minimizes the control overhead by reducing the number of broadcast route discoveries as much as possible using multi-path routing. Also, LPBR-M yields an average hop count per multi-path that is almost equal to that of the minimum-hop based multi-path routing protocols. The rest of the paper is organized as follows: Section 2 reviews the multi-path routing protocols proposed for MANETs. Section 3 provides a detailed design of the LPBR-M protocol. Section 4 describes the simulation environment, defines the performance metrics, presents the simulation results and interprets them. Section 5 concludes the paper. 


\section{Review of Multi-path Routing Protocols for Mobile Ad hoc Networks}

2.1 Split Multi-path Routing Protocol: In Split multi-path routing (Lee and Gerla, 2001), the intermediate nodes forward RREQs that are received along a different link and with a hop count that is not larger than the first received RREQ. The destination selects the route on which it received the first RREQ packet (which will be a shortest delay path), and then waits to receive more RREQs. The destination node then selects the path which is maximally disjoint from the shortest delay path. If more than one maximally disjoint path exists, the tie is broken by choosing the path with the shortest hop count.

2.2 Ad hoc On-demand Multi-path Distance Vector (AOMDV) Routing Protocol: The Ad hoc On-demand Multi-path Distance Vector (AOMDV) routing protocol (Marina and Das, 2001) is an extension of AODV to compute multiple loop-free link-disjoint routes. The RREQs that arrive via different neighbors of the source node define the maximum number of node-disjoint/linkdisjoint paths that are possible. For every destination node $d$, an intermediate node $i$ maintains the list of next hop nodes, the hop count for the different paths to the destination node $d$ and the "advertised hop count"(the maximum hop count for all paths from $i$ to $d$ ), with respect to the latest known sequence number for $d$. An intermediate node accepts and forwards a route advertisement as an alternate path to the destination only if the route advertisement came from a neighbor node that has not yet sent the route advertisement for the destination sequence number and the hop count in the route advertisement is less than the advertised hop count to the destination. When a node receives a route advertisement for the destination with a greater sequence number, the next hop list and the advertised hop count values are reinitialized. The destination node replies for the RREQs arriving from unique neighbors. A multi-path routing scheme that extends AOMDV by using a traffic-path allocation scheme has been proposed (Bononi and Felice, 2006) and it is based on cross-layer measurements of path statistics that reflects the queue size and congestion level of each path. The proposed scheme also utilizes the Fast Forward (FF) MAC forwarding mechanism (Acharya et al., 2002) to reduce the effects of self-contention among frames at the MAC layer.

2.3 AODV-Multi-path (AODVM) Routing Protocol: The AODV-Multi-path (AODVM) routing protocol (Ye et al., 2003) is an extension of the AODV protocol to determine node-disjoint routes. An intermediate node does not discard duplicate RREQ packets and records them in a RREQ table. The destination responds with an RREP for each RREQ packet received. An intermediate node on receiving the RREP, checks its RREQ table and forwards the packet to the neighbor that lies on the shortest path to the source. The neighbor entry is then removed from the RREQ table. Also, whenever a node hears a neighbor node forwarding the RREP packet, the node removes the entry for the neighbor node in its RREQ table.

2.4 Geographic Multi-path Routing Protocol: The Geographic Multi-path Routing Protocol (Loscri and Marano, 2006) has been proposed to reduce interference due to route coupling. The RREQ will have information regarding the locations of the first hop and the last hop intermediate nodes on the path. The destination chooses the path through which it first received the RREQ. For a subsequently received RREQ, the destination measures the distance between the first hops of the path traversed by this RREQ and the already selected paths and also the distance between the last hops of the path traversed by this RREQ and the already selected paths. If both these distances are greater than twice the transmission range of the nodes, the path traversed by the received RREQ is selected.

2.5 Energy-aware Multi-path Routing Protocol: EMRP is an energy-aware multi-path routing protocol (Li et al., 2005) that considers the available energy and the forwarding load at the intermediate nodes of the multiple paths before distributing the load across them. The destination node replies with a RREP packet for each RREQ packet. An intermediate node receiving the RREP packet updates information regarding the distance between the node and the next hop node, the number of retransmission attempts corresponding to the last successful transmission, the current queue length, the current remaining energy of the node. The source node then computes a weight for each route through which the RREP traversed. Routes with minimum weight are preferred as such routes have more remaining energy, less energy consumption due to transmission and reception, less crowded channel in the neighborhood of the nodes in the path and more bandwidth available.

\section{Design of the Node-Disjoint LPBR Multi-path (LPBR-M) Routing Protocol}

3.1 Basic Idea of the Multi-path Extension of LPBR (LPBR-M): The multi-path extension of LPBR works as follows: When a source attempts to send data to the destination and does not know any path to reach the latter, the source broadcasts a Multi-path Route Request (MP-RREQ) message throughout the network. Any broadcast algorithm (for example: flooding or DMEF (Meghanathan, 2009b)) can be used for this purpose. The location and mobility information of the intermediate forwarding nodes are recorded in the MP-RREQ messages as a sequence of Location Update Vectors (LUVs). The destination node receives several MP-RREQs and runs a local node-disjoint path selection algorithm to identify the set of node-disjoint paths, ordered in the increasing order of their hop count. The destination sends out the Multi-path Route Reply (MP-RREP) messages to the source along each of the node-disjoint paths selected. The source receives the MP-RREPs and stores the set of node-disjoint paths (NDPSet) in its local cache. 
For data propagation, the source uses the minimum-hop path in the NDP-Set discovered and continues to use the path until it exists. If an intermediate node could not forward a data packet, it sends a MP-RERR message back to the source. When the source receives the MP-RERR message, it removes the failed path from the NDP-Set and sends the data packet on the next minimum-hop path in the NDP-Set. This procedure is repeated until the source no longer receives a MP-RERR message from an intermediate node or until the NDP-Set is exhausted. In the latter case, the source does not immediately opt for a broadcast discovery procedure. The source waits for the destination to predict a new set of node-disjoint paths based on the LUVs collected in the latest broadcast discovery procedure.

The destination predicts the current location of the nodes and locally constructs a predicted global graph. The node-disjoint path selection heuristic (Meghanathan, 2007) is run on this graph and a set of predicted node-disjoint paths is determined. The destination sends a sequence of MP-LPBR-RREP messages to the source along each of these predicted paths. If a predicted path does not exist, an intermediate node (on the predicted path) cannot forward the MP-LPBR-RREP message further towards the source and instead sends a MP-LPBR-RERR message back to the destination. If the destination receives MP-LPBR-RREP-RERR messages for all the MP-LPBR-RREP messages sent, it discards the LUVs and waits for the source to initiate a new broadcast discovery procedure. If the destination does not receive the MP-LPBR-RREP-RERR message for a particular MP-LPBR-RREP message, it means the corresponding predicted path does actually exist at the current time. If the source receives at least one MPLPBR-RREP message, it stores them the corresponding path in its NDP-Set. For data propagation, the source follows the same procedure of using the paths in its updated NDP-Set in the increasing order of their hop counts. If the source does not receive even one MP-LPBR-RREP message within a certain timeout period, the source then initiates a new broadcast discovery procedure.

3.2 Objectives and Assumptions: The objective of the multi-path extension to LPBR (LPBR-M) is to simultaneously minimize the number of multi-path broadcast discoveries as well as the hop count of the source-destination path. If the broadcast discovery procedure used is the recently proposed Density and Mobility-aware Energy Efficient (DMEF) strategy, we assume the periodic exchange of beacons in the neighborhood of each node at a frequency determined from a time period uniformly and randomly selected from $[0 \ldots 5$ seconds]. We also assume that the clocks across all nodes are synchronized. This is essential to ensure proper timeouts at the nodes for failure to receive a certain control message.

3.3 Broadcast of Multi-path Route Request (MP-RREQ) Messages: Whenever a source node has data packets to send to a destination and is not aware of any path to the latter, the source initiates a broadcast route discovery procedure by broadcasting a Multi-path Route Request (MP-RREQ) message to its neighbors. Any broadcast route discovery procedure (e.g., flooding or DMEF) can be used for this purpose. The source maintains a monotonically increasing sequence number for the broadcast route discoveries it initiates to find the node-disjoint multi-paths. Each node, except the destination, on receiving the first MP-RREQ of the current broadcast process (i.e., a MP-RREQ with a sequence number greater than those seen before), includes its Location Update Vector, LUV, in the MP-RREQ message. The LUV of a node comprises the following: node ID, X, Y co-ordinate information, Current velocity and Angle of movement with respect to the $X$-axis. The node ID is also appended on the "Route Record" field of the MP-RREQ message. The structure of the LUV and the MP-RREQ message is shown in Figures 2 and 3 respectively. Note that upon receiving a MP-RREQ message, we do not let an intermediate node to immediately generate a MPRREP message to the source, even though the intermediate node might know of one or more routes to the destination. We intentionally do this so that we could collect the latest LUVs of each node in the network through the MP-RREQ messages and also able to determine the set of valid of node-disjoint paths that really exist at the time of the broadcast multi-path route discovery process.

\begin{tabular}{|c|c|c|c|c|}
\hline Node ID & X Co-ordinate & Y Co-ordinate & Node Velocity & Angle of Movement \\
\hline 4 bytes & 8 bytes & 8 bytes & 8 bytes & 8 bytes \\
\hline
\end{tabular}

Figure 2. Location Update Vector (LUV) Collected from Each Node

\begin{tabular}{|c|c|c|c|c|}
\hline Source ID & Destination ID & $\begin{array}{c}\text { Sequence } \\
\text { Number }\end{array}$ & $\begin{array}{l}\text { Route Recorded } \\
\text { (List of Node IDs) }\end{array}$ & $\begin{array}{l}\text { Location Update } \\
\text { Vectors (LUVs) }\end{array}$ \\
\hline 4 bytes & 4 bytes & 4 bytes & $\begin{array}{c}\text { Variable Size } \\
\text { of } 4 \text { bytes }\end{array}$ & $\begin{array}{c}\text { Variable Size } \\
\text { of } 36 \text { bytes }\end{array}$ \\
\hline
\end{tabular}

Figure 3. Structure of the Multi-path Route Request (MP-RREQ) Message

3.4 Determination of the Set of Node-Disjoint Paths using the MP-RREQ Messages: When a destination receives a MP-RREQ message, it extracts the path traversed by the message (sequence of Node IDs in the Route Record) and the LUVs of the source and the intermediate nodes that forwarded the message. The destination stores the path information in a set, RREQ-Path-Set, maintained for every source with which the destination is in communication. The paths in the RREQ-Path-Set are stored in the increasing order of their hop count. Ties between paths with the same hop count are broken in the order of their time of arrival at 
the destination node. The LUVs are stored in the LUV-Database maintained for the latest broadcast route discovery procedure initiated by the source. The destination runs a local path selection heuristic to extract the set of node-disjoint paths from the RREQPath-Set. The heuristic makes sure that in the set of node-disjoint paths, except the source and the destination nodes, a node can serve as an intermediate node in at most only one path. A RREQ-ND-Set (set of Node-Disjoint paths) is initialized and updated with the paths extracted from the RREQ-Path-Set satisfying this criterion.

Input: RREQ-Path-Set // set of paths traversed by the MP-RREQ messages received

Output: RREQ-ND-Set // set of node-disjoint paths to be extracted from the RREQ-Path-Set

Initialization: $R R E Q-N D$-Set $\leftarrow \Phi$

Auxiliary Variables: candidatePath // used to store information whether a path extracted from RREQ-Path-Set can be added to RREQ-ND-Set or not

Begin RREQ-ND-Path-Selection

while $(R R E Q-P a t h-S e t \neq \Phi)$ do

Extract the first path $P$ in RREQ-Path-Set // basically removes the path $P$ from RREQ-Path-Set

candidatePath $\leftarrow$ True

for (every intermediate node $u \in P$ ) do

for (every node-disjoint path $N D-P$ in $R R E Q-N D$-Set) do

if ( $u$ is an intermediate node of $N D-P$ ) then

candidatePath $\leftarrow$ False

end if

end for

end for

if (candidatePath is set to True) then

$R R E Q-N D$-Set $\leftarrow R R E Q-N D$-Set $\mathrm{U}\{P\}$

end if

end while

return $R R E Q-N D$-Set

End RREQ-ND-Path-Selection

Figure 4. Heuristic to Extract Node-Disjoint Paths from the MP-RREQ Messages Received

The heuristic (illustrated in Figure 4) traverses through the RREQ-Path-Set in the order of the paths stored in it (in the increasing order of the hop counts). A path $P$ in the RREQ-Path-Set is added to the RREQ-ND-Set only if none of the intermediate nodes in $P$ are already part of any of the paths in the RREQ-ND-Set. Once the RREQ-ND-Set is formed, the destination sends a Multi-path Route Reply (MP-RREP) message for every path in the RREQ-ND-Set. The structure of the MP-RREP message is shown in Figure 5. An intermediate node receiving the MP-RREP message updates its routing table by adding the neighbor that sent the message as the next hop on the path from the source to the destination. The MP-RREP message is then forwarded to the next node towards the source as indicated in the Route Record field of the message.

\begin{tabular}{|c|c|c|c|}
\hline $\begin{array}{l}\text { Originating } \\
\text { Source ID of the } \\
\text { MP-RREQ }\end{array}$ & $\begin{array}{c}\text { Targeted } \\
\text { Destination ID } \\
\text { of the MP-RREQ }\end{array}$ & $\begin{array}{l}\text { Sequence } \\
\text { Number of the } \\
\text { MP-RREQ }\end{array}$ & $\begin{array}{l}\text { Route Recorded } \\
\text { in the MP-RREQ } \\
\text { (List of Node IDs) }\end{array}$ \\
\hline 4 bytes & 4 bytes & 4 bytes & $\begin{array}{c}\text { Variable Size } \\
\text { Multiples of } 4 \text { bytes }\end{array}$ \\
\hline
\end{tabular}

Figure 5. Structure of the MP-RREP Message

3.5 Multi-path Acquisition Time and Data Transmission: After receiving the MP-RREP messages from the destination within a certain time called the Multi-path Acquisition Time (MP-AT), the source stores the paths learnt in a set of node-disjoint paths, NDP-Set. The MP-AT is based on the maximum possible diameter of the network (an input parameter in our simulations). The diameter of the network is the maximum of the hop count of the minimum hop paths between any two nodes in the network. The $M P-A T$ is dynamically set at a node depending on the time it took to receive the first MP-RREP for a broadcast discovery process. If pktOriginInterval denotes the time between the transmission of successive packets from the source, delFirstRREQRecvd indicates the time lapsed between the initiation of the MP-RREQ broadcast and the receipt of the first MP-RREP and hopsFirstRREQRecvd denotes the number of hops traversed by the first MP-RREP received, then, 


$$
M P-A T=\text { Minimum }\left[\text { pktOriginInterval, }\left(\frac{\text { delFirstRREQRecvd }{ }^{*} \text { Diameter }}{\text { hopsFirstRREQRecvd }}\right)\right]
$$

\begin{tabular}{|c|c|c|c|c|c|c|}
\hline $\begin{array}{l}\text { Source } \\
\text { ID }\end{array}$ & $\begin{array}{c}\text { Destination } \\
\text { ID }\end{array}$ & $\begin{array}{l}\text { Sequence } \\
\text { Number }\end{array}$ & $\begin{array}{c}\text { Number of } \\
\text { Disjoint Paths }\end{array}$ & $\begin{array}{c}\text { More } \\
\text { Packets }\end{array}$ & $\begin{array}{c}\text { Current } \\
\text { Dispatch Time }\end{array}$ & $\begin{array}{l}\text { Time Left for } \\
\text { Next Dispatch }\end{array}$ \\
\hline byte & 4 bytes & 4 bytes & 1 byte & 1 bit & 8 bytes & 4 bytes \\
\hline
\end{tabular}

Figure 6. Structure of the Data Packet

When the source begins to start propagating the data packets using the newly formed NDP-Set, the source uses the path with the minimum hop count among the paths in the NDP-Set. The structure of a data packet is illustrated in Figure 6 . The sequence number field in the header can be used by the destination to accumulate and reorder the data packets, incase if they are received out of order. In addition to these regular fields, the header of the data packet includes four specialized fields: the 'Number of Disjoint Paths (NDP-Set Size)' field that indicates the number of active node-disjoint paths currently being stored in the NodeDisjoint Path Set of the source, the 'More Packets' (MP) field, the 'Current Dispatch Time' (CDT) field and the 'Time Left for Next Dispatch' (TNLD) field. The CDT field stores the time as the number of milliseconds lapsed since Jan 1, 1970, 12 AM. These additional overhead (relative to that of the other ad hoc multicast routing protocols) associated with the header of each data packet amounts to only 13 more bytes per data packet.

The source sets the $C D T$ field in all the data packets sent. In addition, if the source has any more data to send, it sets the MP flag to 1 and sets the appropriate value for the TLND field (equal to pktOriginInterval), which indicates the number of milliseconds since the CDT. If the source does not have any more data to send, it will set the MP flag to 0 and leaves the TLND field blank. As we assume the clocks across all nodes are synchronized, the destination node will be able to calculate the end-to-end delay for the data packet based on the time the data packet reaches the node and the $C D T$ field in the header of the data packet. Several clock synchronization algorithms (example: Naumov et al., 2006; Sheu et al., 2007) have been proposed for wireless ad hoc networks. The destination node computes and maintains the average end-to-and delay per data packet for the current path to the source by recording the sum of the end-to-end delays of all the data packets received so far on the path and the number of data packets received on the path. Accordingly, the average end-to-end delay per data packet for the current path is updated every time after receiving a new data packet on the path. If the source node has set the MP flag, the destination node computes the 'Next Expected Packet Arrival Time' (NEPAT), which is CDT field + TLND field $+2 * N D P$-Set Size*Average end-to-end delay per data packet. A timer is started for the NEPAT value. Since, we are using only the average end-to-end delay per data packet to measure the NEPAT value, the variations in the end-to-end delay of particular data packets will not very much affect the NEPAT value. So, the source and destination nodes need not be perfectly synchronized. The clocks across the nodes can have small drifts and this would not very much affect the performance of LPBR-M.

3.6 Multi-path Maintenance: If a link failure occurs due to the two nodes constituting the link drifting away, the upstream node of the broken link (learnt through the failure to successfully transmit the data packet at the link layer) informs about the broken route to the source node through a Multi-path-Route-Error (MP-RERR) message, structure shown in Figure 7. The source node on learning the route failure will remove the failed path from its NDP-Set and attempt to send data packet on the next minimum-hop path in the NDP-Set. If this path is actually available in the network at that time instant, the data packet will successfully propagate its way to the destination. Otherwise, the source receives a MP-RERR message on the broken path, removes the failed path from the NDP-Set and attempts to route the data packet on the next minimum hop path in the NDP-Set. This procedure is repeated until the source does not receive a MP-RERR message or runs out of an available path in the NDP-Set. In the former case, the data packet successfully reaches the destination and the source continues to transmit the next data packet at the next scheduled time. In the latter case, the source is not able to successfully transmit the data packet to the destination.

\begin{tabular}{|c|c|c|c|c|}
\hline \begin{tabular}{|c|} 
Node originating \\
the MP-RERR \\
message
\end{tabular} & $\begin{array}{c}\text { Source ID of } \\
\text { the Data packet } \\
\text { dropped }\end{array}$ & $\begin{array}{c}\text { Destination ID of } \\
\text { the Data packet } \\
\text { dropped }\end{array}$ & $\begin{array}{c}\text { Sequence Number } \\
\text { of the Data packet } \\
\text { dropped }\end{array}$ & $\begin{array}{l}\text { Downstream } \\
\text { Node with which } \\
\text { the link failed }\end{array}$ \\
\hline & & 4 bytes & 4 bytes & yyt \\
\hline
\end{tabular}

Figure 7. Structure of the MP-RERR Message

Before initiating another broadcast route discovery procedure, the source will wait for the destination node to inform it of a new set of node-disjoint routes through a sequence of MP-LPBR-RREP messages. The source will run a MP-LPBR-RREP-timer and wait to receive at least one MP-LPBR-RREP message from the destination. For the failure of the first set of node-disjoint paths, the value of this timer would be a variable parameter within the simulations. In this research work, we will be simulating with 
constant-bit rate (CBR) traffic and so the MP-LPBR-RREP-timer will be set to the route acquisition time (the time it took to get the first MP-RREP message from the destination since the inception of the route discovery), so that we give sufficient time for the destination to learn about the route failure and generate a new sequence of MP-LPBR-RREP messages. For subsequent routerepairs, the MP-LPBR-RREP-timer will be set based on the time it takes to get the first MP-LPBR-RREP message from the destination.

3.7 Prediction of Node Location using the Location Update Vector: If the destination node does not receive the data packet within the NEPAT time, it will attempt to locally construct the global topology using the location and mobility information of the nodes learnt from the latest broadcast route discovery. Each node is assumed to be continuing to move in the same direction with the same speed as mentioned in its latest LUV. Based on this assumption and information from the latest LUVs, the location of each node at the NEPAT time is predicted. Whenever a node changes its direction, we assume the node is moving in the new direction with a particular velocity and towards a particular targeted destination location. As a result, a node can determine its angle of movement with respect to the X-axis at time STIME by computing the slope of the line joining the current location co-ordinates of the node at time STIME and the co-ordinates of the targeted location to which the node is moving. After reaching the targeted location, a node can change its velocity and direction to move to a new destination location.

We now explain how to predict the location of a node (say node $u$ ) at a time instant CTIME based on the LUV gathered from node $u$ at time STIME. Let $\left(X_{u}^{\text {STIME }}, Y_{u}^{\text {STIME }}\right)$ be the $\mathrm{X}$ and $\mathrm{Y}$ co-ordinates of node $u$ at time STIME. Let Angle ${ }_{u}^{\text {STIME }}$ and Velocity $_{u}^{\text {STIME }}$ represent the angle of movement with respect to the X-axis and the velocity at which node $u$ is moving. The distance traveled by node $u$ from time STIME to CTIME would be: Distance ${ }_{u}^{\text {STIME-CTIME }}=(\text { CTIME }- \text { STIME }+1)^{*}$ Velocity ${ }_{u}^{\text {STIME }}$.

Let $\left(X_{u}^{\text {CTIME }}, Y_{u}^{\text {CTIME }}\right)$ be the predicted location of node $u$ at time CTIME. The value of $X_{u}^{\text {CTIME }}$ is given by $X_{u}^{\text {STIME }}+$ Offset$X_{u}{ }^{C T I M E}$ and the value of $Y_{u}^{C T I M E}$ is given by $Y_{u}^{\text {STIME }}+$ Offset- $Y_{u}{ }^{C T I M E}$. The offsets in the X and Y-axes, depend on the angle of movement and the distance traveled, and are calculated as follows:

$$
\begin{aligned}
& \text { Offset- } X_{u}{ }^{\text {CTIME }}=\text { Distance }_{u}^{\text {STIME-CTIME }} * \cos \left(\text { Angle }{ }_{u}^{\text {STIME }}\right) \\
& \text { Offset }-Y_{u}^{\text {CTIME }}=\text { Distance }_{u}^{\text {STIME-CTIME }} * \sin \left(\text { Angle }_{u}{ }^{\text {STIME }}\right) \\
& X_{u}^{\text {CTIME }}=X_{u}^{\text {STIME }}+\text { Offset- } X_{u}^{\text {CTIME }} \\
& Y_{u}^{\text {CTIME }}=Y_{u}^{\text {STIME }}+\text { Offset }-Y_{u}^{\text {CTIME }}
\end{aligned}
$$

We assume each node is initially configured with information regarding the network boundaries, given by [0, 0], $\left[X_{\max }, 0\right]$, $\left[X_{\max }\right.$, $\left.Y_{\max }\right]$ and $\left[0, Y_{\max }\right]$. When the predicted $\mathrm{X}$ and/or $\mathrm{Y}$ co-ordinate is beyond the network boundaries, we set their values to the boundary conditions as stated below.

$$
\begin{aligned}
& \text { If }\left(X_{u}^{C T I M E}<0\right) \text {, then } X_{u}{ }^{C T I M E}=0 ; \quad \text { If }\left(X_{u}^{C T I M E}>X_{\max }\right) \text {, then } X_{u}{ }^{C T I M E}=X_{\max } \\
& \text { If }\left(Y_{u}^{C T I M E}<0\right) \text {, then } Y_{u}^{C T I M E}=0 ; \quad \text { If }\left(Y_{u}^{C T I M E}>Y_{\max }\right) \text {, then } Y_{u}^{C T I M E}=Y_{\max }
\end{aligned}
$$

Based on the predicted locations of each node in the network at time CTIME, the destination node locally constructs the global topology. Note that there exists an edge between two nodes in the locally constructed global topology, if the predicted distance between the two nodes (with the location information obtained from the $L U V$ ) is less than or equal to the transmission range of the nodes.

3.8 LPBR-M: Multi-path Prediction: The destination node locally runs the algorithm for determining the set of node-disjoint paths (Meghanathan, 2007) on the predicted global topology. The algorithm is explained as follows and is illustrated in Figure 8: Let $G$ $(V, E)$ be the graph representing the predicted global topology. Note that $V$ is the set of vertices and $E$ is the set of edges in the predicted network graph. Let the source be identified by $s$ and destination by $d$ and $P_{N}$ denote the set of node-disjoint $s$ - $d$ paths. To start with, we run the $\mathrm{O}\left(n^{2}\right)$ Dijkstra minimum-hop path algorithm on $G$ to determine the minimum hop s- $d$ path in a graph of $n$ nodes. If there is at least one $s$ - $d$ path in $G$, we include the minimum hop $s$ - $d$ path $p$ in the set $P_{N}$. We then remove all the intermediate nodes (nodes other than source $s$ and destination $d$ ) that were part of the minimum-hop $s$ - $d$ path $p$ in the original graph $G$ to obtain the modified graph $G^{\prime}\left(V^{\prime}, E^{\prime}\right)$. We determine the minimum-hop $s$ - $d$ path in the modified graph $G^{\prime}$ ( $\left.V^{\prime}, E^{\prime}\right)$, add it to the set $P_{N}$ and remove the intermediate nodes that were part of this $s$ - $d$ path to get a new updated $G^{\prime}$ ( $V$ ', $E^{\prime}$ ). We repeat this procedure until there exists no more $s-d$ paths in the network. The set $P_{N}$ contains the node-disjoint $s$ - $d$ paths in the original network graph $G$. Note that when we remove a node from a network graph, we also remove all the links associated with the node.

\footnotetext{
Input: Graph $G(V, E)$, source $s$ and destination $d$

Output: Set of node-disjoint paths $P_{N}$

Auxiliary Variables: Graph G', ( $\left.V^{\prime}, E^{\prime \prime}\right)$

Initialization: $G$ ' ' $\left(V^{\prime}, E^{\prime \prime}\right) \leftarrow G(V, E), P_{N} \leftarrow \varphi$.
} 


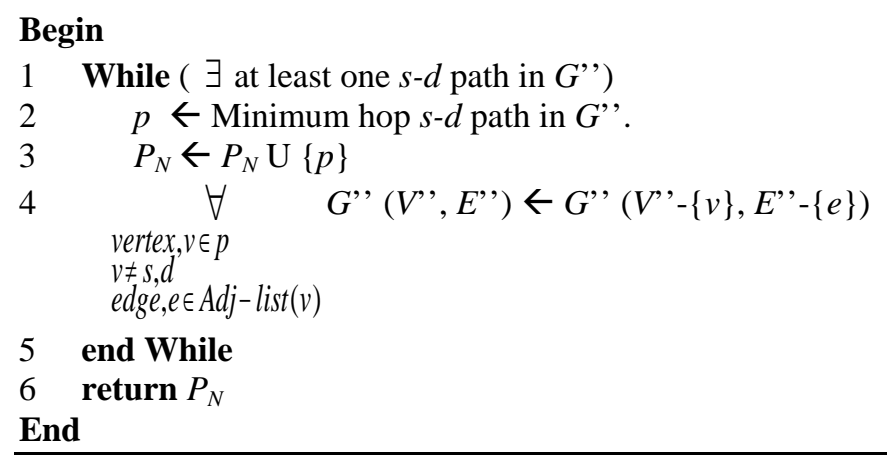

Figure 8. Algorithm to Determine the Set of Node-Disjoint Paths (Meghanathan (2007))

3.9 Propagation of the MP-LPBR-RREP Messages: The destination $d$ sends a MP-LPBR-RREP message to the source $s$ on each of the predicted node-disjoint paths. The intermediate nodes on the discovered path attempt to forward the MP-LPBR-RREP message to the next node on the path to the source node $s$. Each intermediate node receiving the MP-LPBR-RREP message updates its routing table to record the incoming interface of the message as the outgoing interface for any new data packets received from the source $s$ to the destination $d$. The MP-LPBR-RREP message has a "Number of Disjoint Paths' field to indicate the total number of paths predicted and a 'Is Last Path' Boolean field that indicates whether or not the reported path is the last among the set of nodedisjoint paths predicted. If the source node $s$ receives at least one MP-LPBR-RREP message before the MP-LPBR-RREP-timer expires, it indicates that the corresponding predicted $s-d$ path on which the message propagated through, does exists in reality. The source node creates a new instance of the NDP-Set and stores all the newly learnt predicted node-disjoint $s$ - $d$ routes and starts sending data on the minimum hop path among them.

\begin{tabular}{|c|c|c|c|c|c|}
\hline \begin{tabular}{|c|} 
Source \\
Node of \\
the Session
\end{tabular} & $\begin{array}{c}\text { Destination } \\
\text { Node of } \\
\text { the Session }\end{array}$ & $\begin{array}{c}\text { Sequence Number } \\
\text { of the Latest } \\
\text { MP-RREQ }\end{array}$ & $\begin{array}{l}\text { Number of } \\
\text { Disjoint } \\
\text { Paths }\end{array}$ & $\begin{array}{c}\text { Is Last } \\
\text { Path }\end{array}$ & $\begin{array}{l}\text { Predicted Source - } \\
\text { Destination Path } \\
\text { (List of Node IDs) }\end{array}$ \\
\hline $4 b$ & $4 \mathrm{~b}$ & 4 bytes & 1 byte & 11 & $\begin{array}{l}\text { Variable Size: } \\
\text { Multiples of } 4 \text { bytes }\end{array}$ \\
\hline
\end{tabular}

Figure 9. Structure of the MP-LPBR-RREP Message

The source node estimates the Route-Repair Time (RRT) as the time that lapsed between the reception of the last MP-RERR message from an intermediate node and the first MP-LPBR-RREP message from the destination. An average value of the $R R T$ is maintained at the source as it undergoes several route failures and repairs before the next broadcast route discovery. The $M P$ $L P B R$-RREP-timer (initially set to the route acquisition time) will be then set to $1.25^{*}$ Average $R R T$ value, so that we give sufficient time for the destination to learn about the route failure and generate a sequence of MP-LPBR-RREP messages. Nevertheless, this timer value will be still far less than the route acquisition time that would be incurred if the source were to launch a broadcast route discovery. Hence, our approach will only increase the throughput and not decrease it.

3.10 Handling Prediction Failure: If an intermediate node attempting to forward the MP-LPBR-RREP message of the destination could not successfully forward the message to the next node on the path towards the source, the intermediate node informs the absence of the route through a MP-LPBR-RREP-RERR message (structure shown in Figure 10) sent back to the destination. If the destination node receives MP-LPBR-RREP-RERR messages for all the MP-LPBR-RREP messages initiated or the NEPAT time has expired, then the node discards all the LUVs and does not generate any new MP-LPBR-RREP message. The destination node will wait for the source node to initiate a broadcast route discovery. After the MP-LPBR-RREP-timer expires, the source node initiates a new broadcast route discovery.

\begin{tabular}{|c|c|c|c|c|}
\hline $\begin{array}{c}\text { Node originating the } \\
\text { MP-LPBR-RREP-RERR } \\
\text { message }\end{array}$ & $\begin{array}{c}\text { Sequence Number } \\
\text { of MP-LPBR-RREP } \\
\text { packet dropped }\end{array}$ & $\begin{array}{l}\text { Source ID } \\
\text { of the Data } \\
\text { session }\end{array}$ & $\begin{array}{l}\text { Destination } \\
\text { ID of the } \\
\text { Data session }\end{array}$ & $\begin{array}{c}\text { Downstream } \\
\text { Node with which } \\
\text { the link failed }\end{array}$ \\
\hline r & 4 bytes & 4 byte & 4 bytes & 4 bytes \\
\hline
\end{tabular}

Figure 10. Structure of the MP-LPBR-RREP-RERR Message 


\section{Simulations}

The network dimension used is a $1000 \mathrm{~m} \times 1000 \mathrm{~m}$ square network. The transmission range of each node is assumed to be $250 \mathrm{~m}$. The number of nodes used in the network is 25, 50 and 75 nodes representing networks of low, medium and high density with an average distribution of 5, 10 and 15 neighbors per node respectively. Initially, nodes are uniformly randomly distributed in the network. We compare the performance of LPBR-M with that of the link-disjoint routing based Ad hoc On-demand Multi-path Distance Vector (AOMDV) routing protocol and the node-disjoint routing based AODV-Multi-path routing protocol. We implemented all of these three multicast routing protocols in ns-2 (Breslau et al., 2000). The broadcast route discovery strategies simulated are flooding and the density and mobility aware energy-efficient broadcast strategy called DMEF (Meghanathan, 2009b). The simulation parameters are summarized in Table 1.

Table 1. Simulation Conditions

\begin{tabular}{|c|c|c|}
\hline Network Size & \multicolumn{2}{|c|}{$1000 \mathrm{~m} \times 1000 \mathrm{~m}$} \\
\hline Number of nodes & \multicolumn{2}{|c|}{25 (low density), 50 (moderate density) and 75 (high density) } \\
\hline Transmission Range & \multicolumn{2}{|c|}{$250 \mathrm{~m}$} \\
\hline Physical Layer & Signal Propagation Model & Two-ray ground reflection model \\
\hline \multirow{3}{*}{ MAC Layer } & \multicolumn{2}{|c|}{ IEEE 802.11 (Bianchi, 2000) } \\
\hline & Link Bandwidth & $2 \mathrm{Mbps}$ \\
\hline & Interface Queue & FIFO-based, size 200 \\
\hline Routing Protocols & \multicolumn{2}{|l|}{ LPBR-M, AOMDV and AODVM } \\
\hline Broadcast Strategy & \multicolumn{2}{|l|}{ Flooding and DMEF } \\
\hline \multirow{4}{*}{ Mobility Model } & \multicolumn{2}{|c|}{ Random Way Point Model (Bettstetter, 2004) } \\
\hline & Minimum Node Speed, $\mathrm{m} / \mathrm{s}$ & $0 \mathrm{~m} / \mathrm{s}$ \\
\hline & Maximum Node Speed, $\mathrm{m} / \mathrm{s}$ & Low-10; Medium-30; High-50 \\
\hline & Pause Time & 0 second \\
\hline \multirow{4}{*}{ Traffic Model } & \multicolumn{2}{|c|}{ Constant Bit Rate (CBR), UDP } \\
\hline & Number of Source-Destination Pairs & 15 \\
\hline & Data Packet Size & 512 bytes \\
\hline & Packet Sending Rate & 4 Packets/ second \\
\hline \multirow{2}{*}{$\begin{array}{ll}\text { Energy } & \text { Consumption } \\
\text { Model } & \end{array}$} & Transmission Energy & 1.4 W (Feeney, 2001) \\
\hline & Reception Energy & $1 \mathrm{~W}$ (Feeney, 2001) \\
\hline
\end{tabular}

For each combination of network density and node mobility, simulations are conducted with 15 Source-Destination (s- $d$ ) pairs. Traffic sources are constant bit rate (CBR). Data packets are 512 bytes in size and the packet sending rate is 4 data packets/second. Simulation time is 1000 seconds. The node mobility model used is the Random Waypoint model (Bettstetter, 2004). The transmission energy and reception energy per hop is set at $1.4 \mathrm{~W}$ and $1 \mathrm{~W}$ respectively. Initial energy at each node is 1000 Joules.

4.1 Broadcast Strategy - Flooding: Flooding is a widely-used approach for disseminating a message from one node to all the other nodes in a network. In the case of on-demand ad hoc routing protocols, flooding has been also used to discover a path between a pair of nodes in the network, whenever required. For a given network density, flooding offers the highest probability for each node in the network to receive one or more copies of the flooded message. We simulate flooding as follows: The initiating source node sets a monotonically increasing value for the Multi-path Route Request (MP-RREQ) message and broadcasts the message to its complete neighborhood formed by the default maximum transmission range of the node. Each node that receives the MP-RREQ checks if it has received a MP-RREQ with the same or higher sequence number. If so, the received MP-RREQ is simply discarded. Otherwise, the intermediate node inserts its own ID in the Route Record field of the MP-RREQ and broadcasts the message within its complete neighborhood. The destination collects all the MP-RREQ messages and selects the set of node-disjoint paths as explained in the heuristic outlined in Figure 4. A sequence of Multi-path Route Reply (MP-RREP) messages, one on each of the node-disjoint paths, is sent back to the source.

4.2 Broadcast Strategy - DMEF: Meghanathan (2009b) had proposed a density and mobility aware energy-efficient broadcast strategy (called DMEF) to discover long-living stable routes with a reduced energy spent during route discovery. DMEF takes into consideration the number of neighbors of a node (a measure of network density) and node mobility. The average hop count of the routes discovered using DMEF is only at most about $8 \%$ more than that discovered using flooding.

We simulate DMEF as follows for multi-path broadcast route discoveries: The transmission range of a MP-RREQ broadcast is not fixed for every node. A node that is surrounded by more neighbors in the complete neighborhood will broadcast the MP-RREQ only within a smaller neighborhood that would be sufficient enough to pick up the message and forward it to the other nodes in the 
rest of the network. On the other hand, a node that is surrounded by fewer neighbors in the complete neighborhood will broadcast the MP-RREQ to a larger neighborhood (but still contained within the complete neighborhood) so that a majority of the nodes in the complete neighborhood can pick up the message and rebroadcast it further. A node rebroadcasts a MP-RREQ at most once. The density aspect of DMEF thus helps to reduce the unnecessary transmission and reception of broadcast MP-RREQ messages and conserves energy.

To discover stable paths that exist for a longer time, DMEF takes the following approach: A node that is highly mobile makes itself available only to a smaller neighborhood around itself, whereas a node that is less mobile makes itself available over a larger neighborhood (but still contained within the complete neighborhood). The reasoning is that links involving a slow moving node will exist for a long time. Hence, it is better for a slow moving node to advertise itself to a larger neighborhood so that the links (involving this node) that are part of the routes discovered will exist for a longer time. On the other hand, a fast moving node will have links of relatively longer lifetime with neighbors that are closer to it. Hence, it is worth to let a fast moving node advertise only to its nearby neighbors.

The rest of the broadcast process is similar to flooding. The destination node collects all the MP-RREQ messages and selects the set of node-disjoint paths using the heuristic outlined in Figure 4. A sequence of Multi-path Route Reply (MP-RREP) messages, one on each of the node-disjoint paths, is sent back to the source.

4.3 Performance Metrics: The performance metrics studied through this simulation are the following. The performance results for each metric displayed in Figures 11 through 19 are an average of the results obtained from simulations conducted with 5 sets of mobility profiles and 15 randomly picked source-destination $(s-d)$ pairs for each combination of node velocity and network density values.

- Time between Successive Broadcast Multi-path Route Discoveries: This is the time between two successive broadcast multipath route discoveries, averaged over all the $s$ - $d$ sessions over the simulation time. We use a set of multi-paths as long as at least one path in the set exists. We opt for a broadcast route discovery when all the paths in a multi-path set fails. Hence, this metric is a measure of the lifetime of the set of multi-paths and the larger the value of this metric, the better the protocol in terms of multi-path route stability and route discovery control overhead.

- Average Energy Lost per Data Packet Delivered: This is the sum of the energy consumed for transmission and reception at every hop, the energy consumed at the neighbors for coordination during channel access, the energy lost due to route discoveries and the energy lost due to periodic beaconing, if any, averaged over all the data packets delivered successfully from the source to the destination.

- Packet Delivery Ratio: This is the ratio of the total number of data packets delivered to the destination to that of the total number of data packets originating from the source, averaged over all the $s-d$ sessions. With a larger queue size of 200 at each node, the packet delivery ratio is more a representative of the connectivity of the network.

- Energy Lost per Broadcast Multi-path Route Discovery: This is the energy consumed per global broadcast based route discovery attempt, averaged over all the $s-d$ sessions over the entire simulation time. The energy consumed per global broadcast route discovery attempt includes the energy consumed to transmit (broadcast) a MP-RREQ message to all the nodes in the neighborhood and the energy consumed to receive the MP-RREQ message sent by each node in the neighborhood, summed over all the nodes.

- Control Message Overhead: This is the ratio of the total number of control messages (MP-RREQ, MP-RREP, MP-LPBRRREP and MP-LPBR-RREP-RERR) received at every node to that of the total number of data packets delivered at a destination, averaged over all the $s-d$ sessions across the entire simulation time. Note that we prefer to consider the number of control messages received rather than transmitted because, in a typical broadcast operation, the total amount of energy spent to receive a control message at all the nodes in a neighborhood is greater than the amount of energy spent to transmit the message.

- Average Energy Lost per Node: The is the energy lost at a node due to transmission and reception of data packets, control packets and beacons, if any, averaged over all the nodes in the network for the entire simulation time.

- Average Number of Disjoint Paths Found per Multi-path: This is the number of disjoint-paths (link-disjoint or node-disjoint, depending on the routing protocol) determined during a multi-path broadcast route discovery, averaged over all $s-d$ sessions and over the entire simulation time.

- Average Number of Disjoint Paths used per Multi-path: This is the number of disjoint-paths (link-disjoint or node-disjoint, depending on the routing protocol) actually used by the routing protocol, averaged over all the $s$ - $d$ sessions across the entire simulation time. All the disjoint-paths determined during a broadcast route discovery may not be actually used by a routing protocol. Some of the disjoint paths might have failed before the routing protocol considers using them. Note that we use the disjoint paths in the order of their hop count.

- Average Hop Count of all Disjoint-paths used: This is the time-averaged hop count of the disjoint paths determined and used by each of the multi-path routing protocols studied. For example, if a protocol determines the multi-path set $M P_{1}$ and $M P_{2}$ : $M P_{1}$ has three disjoint paths $P_{1-1}, P_{1-2}$ and $P_{1-3}$ with hop count 3, 4 and 2 and are used for 2, 8 and 3 seconds respectively; 
$M P_{2}$ has two disjoint paths $P_{2-1}$ and $P_{2-2}$ with hop count 5 and 3 and are used for 7 and 4 seconds respectively. The timeaveraged hop count of the disjoint paths used is 3.79 and is calculated as follows:

$$
\begin{aligned}
& \text { hopCount }=\frac{\sum_{i=1} \sum_{j=1}^{\text {Multi-Paths \#Paths }[i]}\left[\operatorname{hops}\left(P_{i-j}\right) * \operatorname{time}\left(P_{i-j}\right)\right]}{\text { \#Multi-Paths\#Paths[i]} \sum_{i=1} \sum_{j=1} \text { time }\left(P_{i-j}\right)} \\
& \text { hopCount }=\frac{[3 * 2+4 * 8+2 * 3]+[5 * 7+3 * 4]}{[2+8+3+7+4]}=\frac{91}{24}=3.79
\end{aligned}
$$

4.4 Time between Successive Broadcast Multi-path Route Discoveries: The LPBR-M protocol yields the longest time between successive broadcast multi-path route discoveries (refer Figure 11). This implies that the set of node-disjoint paths discovered and predicted by LPBR-M are relatively more stable than the set of link-disjoint and node-disjoint paths discovered by the AOMDV and AODVM routing protocols respectively. Also, when DMEF is used as the route discovery strategy, each of the three multipath routing protocols yielded a longer time between route discoveries, compared to the use of flooding as the route discovery strategy.

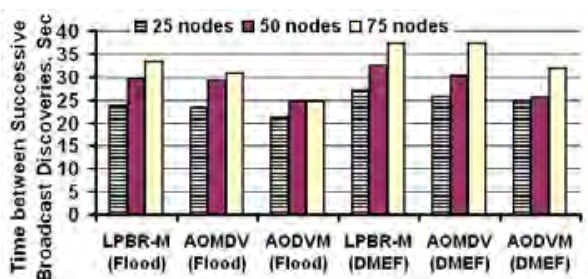

Figure 11.1. $v_{\max }=10 \mathrm{~m} / \mathrm{s}$

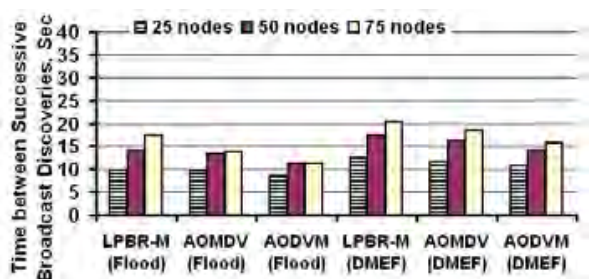

Figure 11.2. $v_{\max }=30 \mathrm{~m} / \mathrm{s}$

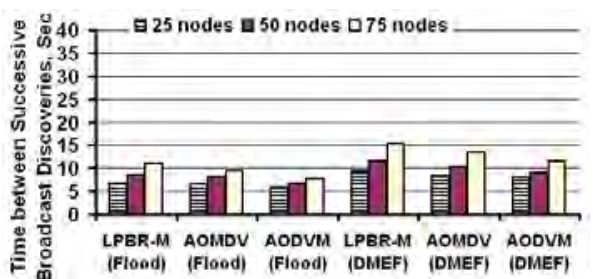

Figure 11.3. $v_{\max }=50 \mathrm{~m} / \mathrm{s}$

Figure 11. Time between Successive Broadcast Multi-path Route Discoveries

As we increase the level of node mobility from low to moderate and high, the difference in the time between successive route discoveries incurred for AOMDV and AODVM vis-à-vis LPBR-M increases. Also, for a given level of node mobility, as we increase the network density from low to moderate and high, the time between successive route discoveries for LPBR-M increases relatively faster compared to those incurred for AOMDV and AODV-M. LPBR-M yields 3\%-17\% and 15\%-44\% more time between successive route discoveries compared to AOMDV and AODVM respectively. For each of the three multi-path routing protocols, the increase in the time between route discoveries when DMEF is used as the route discovery strategy is $4 \%-28 \%, 16 \%$ $38 \%$ and $28 \%-50 \%$ more than that incurred with flooding at low, moderate and high node mobility levels respectively.

4.5 Average Energy Lost per Data Packet Delivered: For a given level of node mobility and network density, the energy consumed per data packet (refer Figure 12) for each of three multi-path routing protocols is not very different from each other (the difference is within 3\%). However, the energy consumed per data packet at a moderate network density of 50 nodes and a high network density of 75 nodes is respectively about $31 \%-44 \%$ and $75 \%-100 \%$ more than the energy consumed per data packet incurred in a low network density of 25 nodes. This can be attributed to the increase in the number of nodes receiving a broadcast message and transmitting the message in the network. Also, more neighbors are involved in the Request-to-Send and Clear-to-Send message reception during co-ordination for channel access in every hop of a path taken by every data packet. In networks with high level of node mobility, we observe that the energy consumed per data packet with flooding as the route discovery strategy can be $2 \%$ (low density)-11\% (high density) more than that obtained with DMEF as the route discovery strategy.

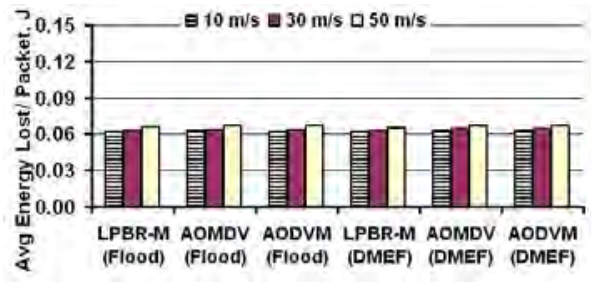

Figure 12.1. 25 Nodes

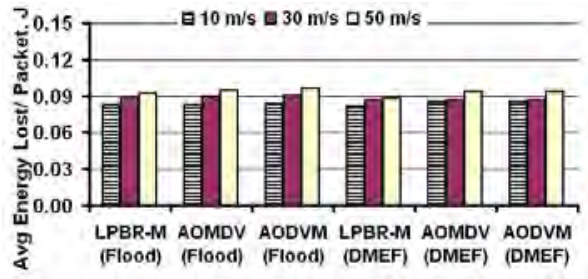

Figure 12.2. 50 Nodes

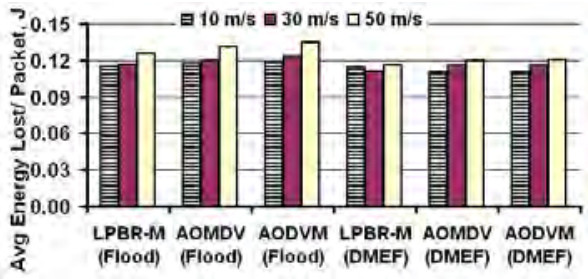

Figure 12.3. 75 Nodes

Figure 12. Average Energy Lost per Data Packet Delivered 
4.6 Packet Delivery Ratio: For a given level of node mobility and network density, the packet delivery ratio (refer Figure 13) of each of the multi-path routing protocols almost remained the same. In networks of low density, we observe $86 \%-93 \%$ packet delivery ratio. Also, in low density networks, we observe that as the level of node mobility increases from low to moderate and high, the packet delivery ratio decreases by about 4\%-5\%. With a FIFO-based queue of size 200 at each node, the lower packet delivery ratio in low-density networks is mainly attributed to poor network connectivity. In moderate and high density networks, each of the three routing protocols yield a packet delivery ratio of at least $98 \%$ and $99 \%$ respectively.

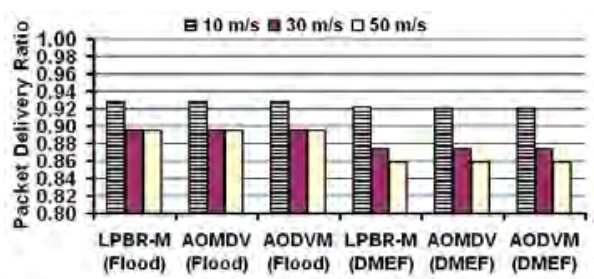

Figure 13.1. 25 Nodes

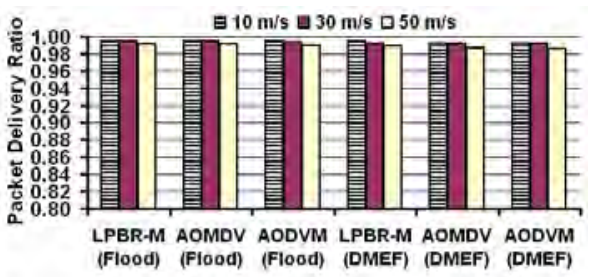

Figure 13.2. 50 Nodes

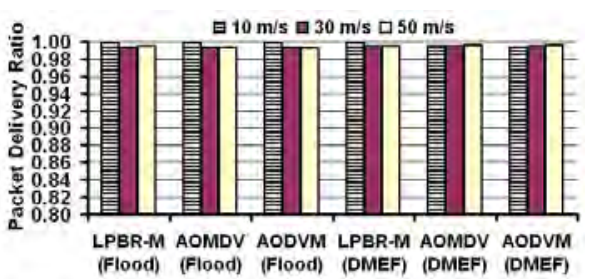

Figure 13.3. 75 Nodes

Figure 13. Packet Delivery Ratio of LPBR-M, AOMDV and AODVM under both Flooding and DMEF

4.7 Energy Lost per Broadcast Multi-path Route Discovery: For a given level of node mobility and network density, the energy consumed per broadcast multi-path route discovery (refer Figure 14) for each of the three multi-path routing protocols is almost the same as this metric depends only on the route discovery strategy and not on the routing protocol. The energy consumed per route discovery in a moderate network density of 50 nodes and a high network density of 75 nodes is respectively about 3.4 to 4.1 times and 8.0 to 8.5 times more than the energy consumed per route discovery in a low network density of 25 nodes. This can be attributed to the increase in the number of nodes receiving a broadcast message and transmitting the message in the network. With the DMEF strategy, we observe a decrease in the magnitude of energy consumed per route discovery at high network density and high node mobility. This can be attributed to the clever adaptation of the broadcast range by the DMEF strategy. In networks of low and moderate density, flooding consumes $19 \%-23 \%$ more energy per route discovery when compared to DMEF; whereas in high density networks, flooding consumes 32-38\% more energy per route discovery compared to DMEF.

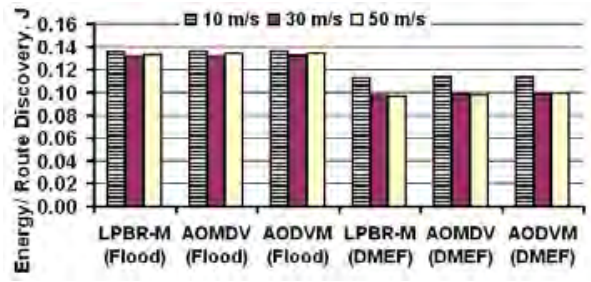

Figure 14.1. 25 Nodes

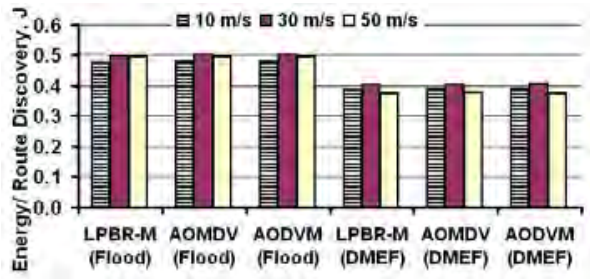

Figure 14.2. 50 Nodes

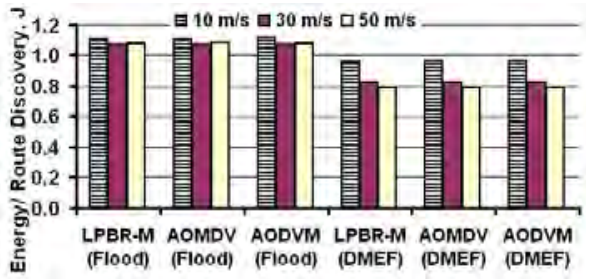

Figure 14.3. 75 Nodes

Figure 14. Energy Lost per Broadcast Route Discovery under both Flooding and DMEF

4.8 Control Message Overhead: For a given level of node mobility and network density, LPBR-M incurs the lowest control message overhead (refer Figure 15). For a given level of node mobility, AOMDV and AODVM respectively incur 4\%-16\% and 14\%-34\% more control message overhead than LPBR-M when flooding is used as the route discovery strategy. On the other hand, when DMEF is used as the route discovery strategy, AOMDV and AODVM respectively incur $10 \%-14 \%$ and $11 \%-23 \%$ more control message overhead than LPBR-M. For a given level of network density, the control message overhead incurred by each of the three routing protocols using flooding as the route discovery strategy in networks of low, moderate and high node mobility is respectively 7\%-39\%, 32\%-58\% and 49\%-110\% more than that incurred with DMEF as the route discovery strategy.

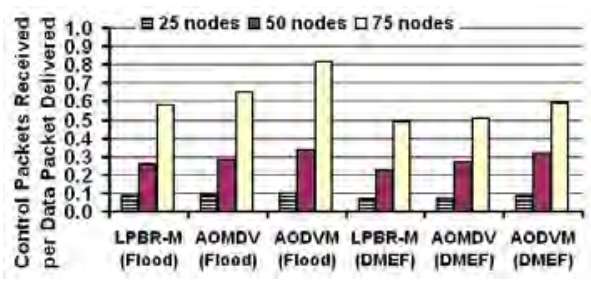

Figure 15.1. 25 Nodes

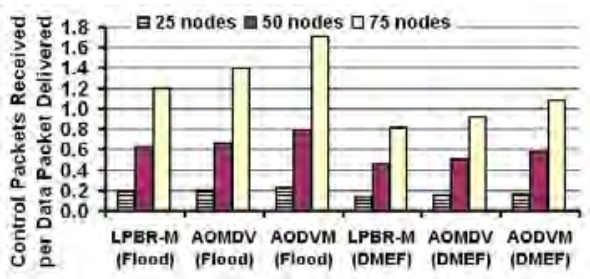

Figure 15.2. 50 Nodes

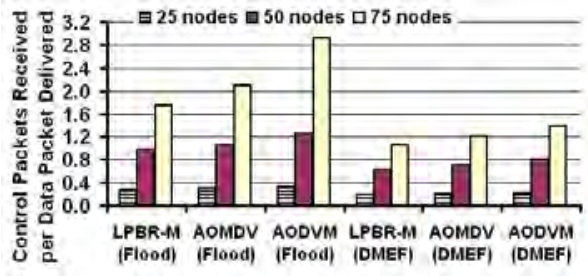

Figure 15.3. 75 Nodes

Figure 15. Control Message Overhead for LPBR-M, AMDV and AODVM under Flooding and DMEF 
In networks of moderate node mobility, the control message overhead incurred by each of the three multi-path routing protocols while using flooding and DMEF is respectively 2.1 (high density) to 3.4 (low density) times and 1.7 to 2.0 times more than that incurred in networks of low node mobility. In networks of high node mobility, the control message incurred by each of the three multi-path routing protocols while using flooding and DMEF is respectively 3.0 (high density) to 3.7 (low density) times and 2.2 (high density) to 2.8 (low density) times more than that incurred in high density networks, the control message overhead incurred in networks of low node mobility. Thus, DMEF substantially reduces the control message overhead as we increase the network density and/or the level of node mobility.

4.9 Average Energy Lost per Node: We conduct all of our simulations with a fixed offered traffic load comprising of $15 \mathrm{~s}-d$ pairs. Hence, as we increase the network density, the net energy consumed per node decreases as more nodes are available in the network for data transfer. For both flooding and DMEF, the energy lost per node in networks of moderate and high density is respectively about $65 \%-75 \%$ and $70 \%-84 \%$ of the energy lost per node in networks of low mobility. For a given network density, the energy lost per node at high node mobility is greater than the energy lost per node at low node mobility by at most $16 \%$ and $10 \%$ when operated with flooding and DMEF respectively.

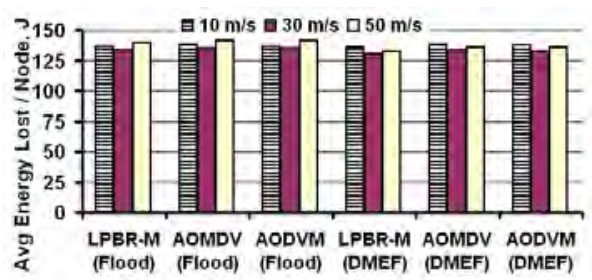

Figure 16.1: 25 Nodes

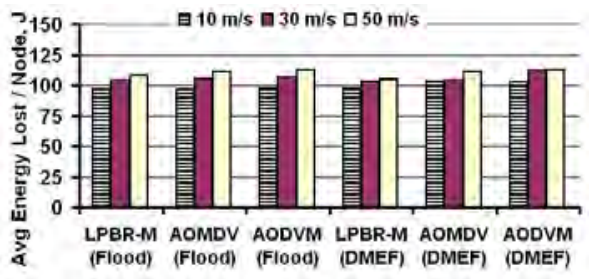

Figure 16.2: 50 Nodes

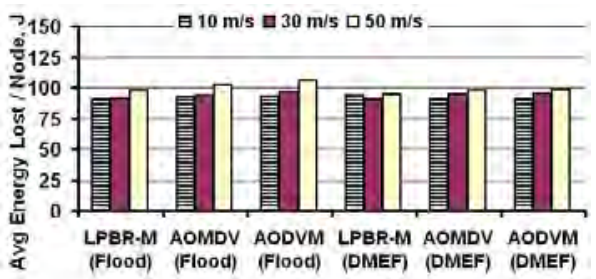

Figure 16.3: 75 Nodes

Figure 16: Average Energy Lost per Node

4.10 Average Number of Disjoint Paths Found per Multi-path: For a given routing protocol and network density, the average number of disjoint paths discovered per multi-path (refer Figure 17) almost remains the same, irrespective of node mobility. With increase in network density, the number of link-disjoint and node-disjoint paths between a source and destination increases. For a given network density and broadcast strategy, the link-disjoint path routing based AOMDV determines a larger number of disjoint paths (32\%-62\% more) than LPBR-M and AODVM; the node-disjoint path routing based LPBR-M determines relatively larger number of disjoint paths (12\%-22\% more) than the other node-disjoint path routing based AODVM. For each of the three routing protocols, the average number of disjoint paths determined in a moderate density network and high-density network is respectively about 55\%-95\% and 120\%-200\% more than that determined in a low-density network. As DMEF reduces the control overhead and the number of nodes forwarding the MP-RREQ messages, the average number of disjoint paths determined for the three routing protocols is about $5 \%$ (low density) to $20 \%$ (high density) lower than that discovered using flooding.

4.11 Average Number of Disjoint Paths used per Multi-path: For a given level of node mobility and network density, the linkdisjoint path based AOMDV had the largest number of disjoint paths actually used. But, the magnitude of the number of AOMDV link-disjoint paths actually used (refer Figure 18) is only at most 25\% more than the number of LPBR-M node-disjoint paths or the AODVM node-disjoint paths. Even though AOMDV had a relatively larger number of link-disjoint paths (as explained in Section 4.8), the percentage of such paths successfully used is the lowest among the three multi-path routing protocols. The node-disjoint path based AODVM routing protocol has the largest percentage of the discovered disjoint paths actually being used. The percentage of node-disjoint paths successfully used in the case of LPBR-M is in between to those of AODVM and AOMDV. As the network density increases, the number of disjoint paths actually used by each of the three multi-path routing protocols increases, nevertheless at a significantly reduced rate. As a result, the percentage of the discovered disjoint paths successfully used decreases with increase in network density. This can be attributed to the failure of the disjoint paths over time and the disjointpaths discovered are not actually available when the routing protocol wants to use them.

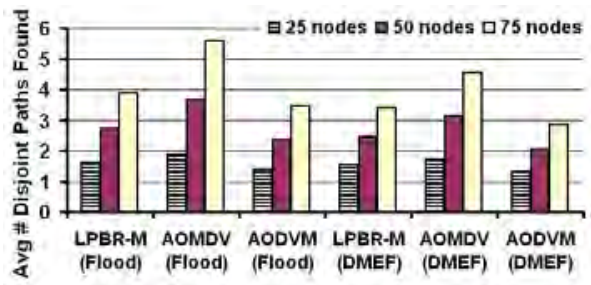

Figure 17. Average Number of Disjoint Paths Found per Multi-path

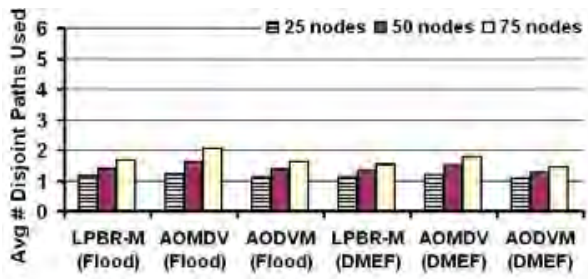

Figure 18. Average Number of Disjoint Paths Used per Multi-path

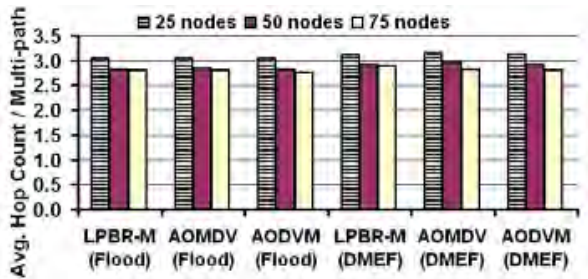

Figure 19. Average Hop Count of All Disjoint Paths Used 
4.12 Average Hop Count of All Disjoint-Paths Used: For a given routing protocol and network density, the average hop count (refer Figure 19) of the disjoint-paths used is almost the same, irrespective of the level of node mobility. As we add more nodes in the network, the hop count of the paths tends to decrease as the source manages to reach the destination through a relatively lesser number of intermediate nodes. With increase in network density, there are several candidates to act as intermediate nodes on a path. The average hop count of the paths in high and moderate density networks is $6 \%-10 \%$ less than the average hop count of the paths in networks of low density. For each of the routing protocols, for all network densities, the average hop count of the paths discovered using DMEF is at most $4 \%$ more than the hop count of the paths determined using flooding.

\section{Conclusions and Future Work}

The high-level contribution of this paper is the design and development of a node-disjoint multi-path extension for the Location Prediction Based Routing protocol (referred to as LPBR-M). LPBR-M has been proposed to reduce the number of global broadcast multi-path route discoveries. Simulations have been conducted with both flooding and DMEF as the broadcast route discovery strategies. We compared the performance of LPBR-M with that of the link-disjoint path based AOMDV and the node-disjoint path based AODVM multi-path routing protocols. LPBR-M achieves the longest time between successive route discoveries and the lowest control message overhead. Also, the LPBR-M multi-paths incur hop count that is very much equal to those obtained with the minimum-hop based AODVM and AODVM routing protocols. Moreover, DMEF helps each of the multi-path routing protocols to determine a set of node or link disjoint paths that exist for a long time and at the same time does not increase the source-destination hop count appreciably. When used with DMEF, each of the multi-path routing protocols incurred a lower energy spent per route discovery, compared to flooding. As future work, we would evaluate the performance of LPBR-M under different mobility models and also compare its performance with that of energy-aware multi-path routing protocols for MANETs.

\section{References}

Acharya, A., Misra, A. and Bansal, S. 2002. A label-switching packet forwarding architecture for multi-hop wireless LANs. Proceedings of the $5^{\text {th }}$ ACM International Workshop on Wireless Mobile Multimedia, pp. 33-40.

Bettstetter, C., Hartenstein, H. and Perez-Costa, X. 2004. Stochastic Properties of the Random-Waypoint Mobility Model. Wireless Networks, Vol. 10, No. 5, pp. 555-567.

Bianchi, G. 2000. Performance Analysis of the IEEE 802.11 Distributed Coordination Function. IEEE Journal of Selected Areas in Communication, Vol. 18, No. 3, pp. 535-547.

Bononi, L. and Felice, M. D. 2006. Performance Analysis of Cross-layered Multi-path Routing and MAC Layer Solutions for Multi-hop Ad Hoc Networks. Proceedings of the ACM International Workshop on Mobility Management and Wireless Access, pp. 190-197.

Breslau, L., Estrin, D., Fall, K., Floyd, S., Heidemann, J., Helmy, A., Huang, P., McCanne, S., Varadhan, K., Xu, Y. and Yu, H. 2000. Advances in Network Simulation. IEEE Computer, Vol. 33, No. 5, pp. 59-67.

Cormen, T. H., Leiserson, C. E., Rivest, R. L. and Stein, C. 2001. Introduction to Algorithms. $2^{\text {nd }}$ Edition, MIT Press/ McGraw Hill, New York.

Feeney, L. M. 2001. An Energy Consumption Model for Performance Analysis of Routing Protocols for Mobile Ad Hoc Networks. Journal of Mobile Networks and Applications, Vol. 3, No. 6, pp. 239-249.

Johnson, D. B., Maltz, D. A., and Broch, J. 2000. DSR: The Dynamic Source Routing Protocol for Multi-hop Wireless Ad hoc Networks. Ad hoc Networking, Chapter 5, Addison Wesley, pp. 139-172.

Lee, S., and Gerla, M. 2001. Split Multi-path Routing with Maximally Disjoint Paths in Ad Hoc Networks. Proceedings of IEEE International Conference on Communications, Vol. 10, pp. 3201-3205.

Li, M., Zhang, L., Li, V. O. K., Shan, X., and Ren, Y. 2005. An Energy-Aware Multi-path Routing Protocol for Mobile Ad Hoc Networks. Proceedings of the ACM SIGCOMM Asia.

Loscri, V. and Marano, S. 2006. A New Geographic Multi-path Protocol for Ad Hoc Networks to Reduce the Route Coupling Phenomenon. Proceedings of the $63^{\text {rd }}$ IEEE Vehicular Technology Conference, VTC 2006-Spring, Vol. 3, pp. 1102-1106.

Marina, M. K. and Das, S. R. 2001. On-demand Multi-path Distance Vector Routing in Ad Hoc Networks. Proceedings of the IEEE International Conference on Network Protocols, pp. 14-23.

Meghanathan, N. 2007. Stability and Hop Count of Node-Disjoint and Link-Disjoint Multi-path Routes in Ad Hoc Networks. Proceedings of the $3^{\text {rd }}$ IEEE International Conference on Wireless and Mobile Computing, Networking and Communications.

Meghanathan, N. 2009a. A Location Prediction-Based Reactive Routing Protocol to Minimize the Number of Route Discoveries and Hop Count per Path in Mobile Ad Hoc Networks. The Computer Journal, Vol. 52, No. 4, pp. 461-482.

Meghanathan, N. 2009b. A Density and Mobility Aware Energy-Efficient Broadcast Strategy to Determine Stable Routes in Mobile Ad hoc Networks. Proceedings of the International Conference on Wireless Networks, pp. 167-173.

Mueller, S., Tsang, R. P. and Ghosal, D. 2004. Multi-path Routing in Mobile Ad Hoc Networks: Issues and Challenges. Lecture Notes in Computer Science, Vol. 2965, pp. 209-234. 
Naumov, V., Baumann, R. and Gross, T. 2006. An Evaluation of Inter-Vehicle Ad hoc Networks based on Realistic Vehicular Traces. Proceedings of the $7^{\text {th }}$ ACM International Symposium on Mobile Ad hoc Networking and Computing, pp. 108-119.

Perkins, C. E. and Royer, E. M. 1999. Ad hoc On-demand Distance Vector Routing. Proceedings of the 2nd Annual IEEE International Workshop on Mobile Computing Systems and Applications, pp. 90-100.

Sheu, J-P., Chao, C-M., Hu, W-K. and Sun, C-W. 2007. A Clock Synchronization Algorithm for Multi-Hop Wireless Ad Hoc Networks. Wireless Personal Communications: An International Journal, Vol. 43, No. 2, pp. 185-200.

Wang, H., Ma, K. and Yu, N. 2005. Performance Analysis of Multi-path Routing in Wireless Ad Hoc Networks. Proceedings of the International Conference on Wireless Communications, Networking and Mobile Computing, Vol. 2, pp. $723-726$.

Xu, W., Yan, P., and Xia, D. 2005. Similar Node-Disjoint Multi-paths Routing in Wireless Ad Hoc Networks. Proceedings of the International Conference on Wireless Communications, Networking and Mobile Computing, Vol. 2, pp. 731-734.

Ye, Z., Krishnamurthy, S. V. and Tripathi, S. K. 2003. A Framework for Reliable Routing in Mobile Ad Hoc Networks. Proceedings of the IEEE International Conference on Computer Communications.

Biographical notes

Dr. Natarajan Meghanathan is currently working as Assistant Professor of Computer Science at Jackson State University, Mississippi, USA, since August 2005. Dr. Meghanathan received his MS and PhD in Computer Science from Auburn University, AL and The University of Texas at Dallas in August 2002 and May 2005 respectively. Dr. Meghanathan's main areas of research interests are wireless ad hoc networks and sensor networks. He has more than 60 peer-reviewed publications in leading international journals and conferences in this area. Dr. Meghanathan has recently received grants from the U. S. Army Research Laboratory (ARL) and National Science Foundation (NSF). Besides ad hoc networks, Dr. Meghanathan is currently conducting active research in the areas of graph theory, network security and bioinformatics. Dr. Meghanathan has organized workshops and conference sessions in the areas of ad hoc networks, parallel and distributed computing. He serves as the editor of a number of international journals and also in the program committee and organization committees of several leading international conferences in the area of networks. He is listed in the Marquis’ Who is Who in America for 2009.

Received June 2010

Accepted June 2010

Final acceptance in revised form June 2010 